Article

\title{
Insecticidal Activity and Insecticidal Mechanism of Total Saponins from Camellia oleifera
}

\author{
Chuanjian Cui ${ }^{\dagger}$, Yunqin Yang ${ }^{\dagger}$, Tianyu Zhao, Kangkang Zou, Chuanyi Peng, Huimei Cai, \\ Xiaochun Wan and Ruyan Hou *D
}

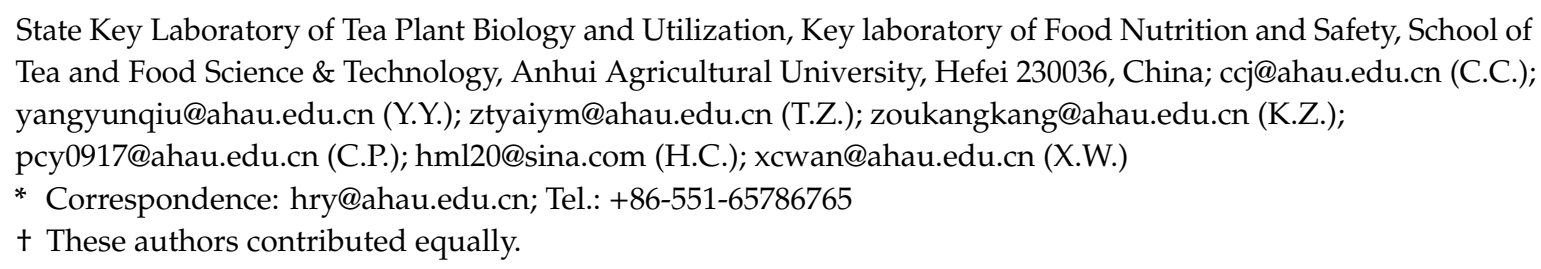
Tea and Food Science \& Technology, Anhui Agricultural University, Hefei 230036, China; ccj@ahau.edu.cn (C.C.); yangyunqiu@ahau.edu.cn (Y.Y.); ztyaiym@ahau.edu.cn (T.Z.); zoukangkang@ahau.edu.cn (K.Z.); pcy0917@ahau.edu.cn (C.P.); hml20@sina.com (H.C.); xcwan@ahau.edu.cn (X.W.)

* Correspondence: hry@ahau.edu.cn; Tel.: +86-551-65786765

† These authors contributed equally.

Academic Editor: Vincenzo De Feo

check for Received: 3 November 2019; Accepted: 8 December 2019; Published: 10 December 2019

\begin{abstract}
Chemical pesticides are commonly used during the cultivation of agricultural products to control pests and diseases. Excessive use of traditional pesticides can cause environmental and human health risks. There are ongoing searches for new plant-derived pesticides to reduce the use of chemical pesticides. In this study, tea saponin extracts of different purities were extracted from Camellia oleifera seeds using AB-8 macroporous resin and gradient elution with ethanol. The insecticidal effects of the tea saponin extracts were evaluated by contact toxicity tests and stomach toxicity tests using the lepidopteran pest of tea plantation, Ectropis obliqua. The total saponins extracted using 70\% ethanol showed strong contact toxicity $\left(\mathrm{LC}_{50}=8.459 \mathrm{mg} / \mathrm{L}\right)$ and stomach toxicity $\left(\mathrm{LC}_{50}=22.395 \mathrm{mg} / \mathrm{L}\right)$. In-depth mechanistic studies demonstrated that tea saponins can disrupt the waxy layer of the epidermis, causing serious loss of water, and can penetrate the inside of the intestine of E. obliqua. After consumption of the tea saponins, the intestinal villi were shortened and the cavities of the intestinal wall were disrupted, which resulted in larval death. This study highlights the potential of tea saponins as a natural, plant-derived pesticide for the management of plant pests.
\end{abstract}

Keywords: tea saponin; Camellia oleifera; Ectropis obliqua; contact toxicity; stomach toxicity; waxy layer; insecticidal mechanism

\section{Introduction}

Tea is one of the most popular non-alcoholic beverages in the world today, favored for its unique aroma and taste [1]. Ectropis obliqua Prout (Lepidoptera:Geometridae) is a chewing defoliator in tea plantations, causing devastating effects on the quality, yield, and growth of tea plants [2,3]. Because of its fast breeding, rapid spatial spread, and large appetite, outbreaks of this insect have resulted in losses of more than $60 \%$ in tea production [4]. E. obliqua is the primary target pest in tea cultivation [5].

At present, E. obliqua is mainly controlled by chemical pesticide spraying [6]. Tea is a direct receptor of aerial pesticides, and pesticide residues may cause food safety and other issues. At the same time, studies have shown that only a small amount (about $0.3 \%$ ) of pesticides can enter the target organism and that most pesticides $(99.7 \%)$ will eventually enter the environment, posing environmental safety hazards [7]. International trade standards demand higher quality agricultural products and set maximum residual levels of pesticides in tea products. Plant-derived products can be more environmentally friendly and can represent cost-effective alternatives to control phytophagous insects and plant-pathogenic microorganisms. Therefore, biological control of this pest has become the method of choice [5]. 
Tea saponins (TS) are a promising bio-pesticide with good dispersibility, permeability and wetting effect [8]. Tea saponin content in the seeds of Camellia oleifera is greater than $10 \%$. The annual output of C. oleifera has exceeded 2 million tons, and about 200,000 tons of saponins can be supplied for use [9]. Previous studies have found that tea saponins have a strong insecticidal effect on the diamondback moth, Plutella xylostella, and aphid, Aphis craccivora [10]. In a study on saponin repellent activity, saponins at $4000 \mathrm{mg} / \mathrm{L}$ highly repel $(48.57 \%)$ the third instar of $P$. xylostella. However, the feeding preference index (PI) of the third instar for saponins was less at higher concentrations (0.63). Treatment of diamondback moth larvae with $\mathrm{LC}_{20}$ and $\mathrm{LC}_{50}$ doses of TS led to lower growth rates, decreased feed consumption, reduced frass production, lower pupal weights, reduced percentage pupation, slower adult emergence, and diminished fecundity, but prolonged durations of the larval and pupal periods [10].

Most current research indicates that the insecticidal mechanism of tea saponins is related to effects on the detoxification enzymes of insects. Tea saponins can reduce superoxide dismutase (SOD), catalase (CAT), acetylcholinesterase (AChE), and carboxylesterase (CES) activities [11,12]. Some studies suggest that the insecticidal activity of saponins is due to their interaction with cholesterol, which leads to interference with the synthesis of ecdysteroids. These substances are also protease inhibitors or cytotoxic to certain insects [13]. However, the specific insecticidal mechanism has not been reported.

In this paper, we isolated saponin extracts from C. oleifera seeds for the purpose of developing new plant-derived pesticides. Using the common pest E. obliqua as a model, the insecticidal effect of tea saponins was comprehensively evaluated by contact toxicity and stomach toxicity tests. The insecticidal mechanism of tea saponins against E. obliqua larvae was studied microscopically, and the source of its insecticidal activity was found.

\section{Results}

\subsection{Saponins in Aqueous Ethanolic Extracts}

Tea saponins were extracted from Camellia oleifera seeds using ethanol and then purified using AB-8 macroporous resin and a gradient ethanol elution. The obtained eluates were subjected to thin-layer chromatography in combination with a sulfuric-acid-ethanol color reaction (Figure S1). The purple color indicating saponins was observed in different eluents. Of all the eluates, $70 \%$ ethanol eluate (EE) did not have a chromogenic reaction of flavonoids, which demonstrates the higher purity of the saponin in the $70 \%$ ethanol eluate.

The extracted tea saponins were analyzed by HPLC (Figure S2). Since there are no commercial Camellia saponin standards, the total saponin content was quantified using an individual oleiferasaponin C1 standard, as described previously [14]. The oleiferasaponin C1 standard curve was expressed as $y=0.6938 x-1.5247\left(R^{2}=0.9999\right)$, showing a linear correlation over the concentration range of 0.005 to $1.0 \mathrm{mg} / \mathrm{mL}$.

The purity of the saponins extracted using different ethanol-water eluents was tested using a standard multitest method. The purity of the saponins in the $C$. oleifera seed cake ethanol extract (CSCEE) was $39.5 \% \pm 3.46 \%$; in the $30 \% \mathrm{EE}$, the purity was $35.9 \% \pm 2.31 \%$; in the $50 \% \mathrm{EE}$, the purity was $55.6 \% \pm 1.69 \%$; in the $70 \% \mathrm{EE}$, the purity was $99 \% \pm 0.71 \%$.

UPLC-Q-TOF/MS analysis was used to characterize the saponins in the 70\% EE. The eluate contained 29 triterpenoid saponins (Figure S3). The molecular formulas and molecular weights of these saponins were confirmed by high-resolution mass measurements, deprotonated molecular ions $[\mathrm{M}-\mathrm{H}]^{-}$, isotope abundance for each pseudomolecular ion, and fragment ions (Tables S1 and S2). The identification of each saponin was based on published fragmentation data and nominal masses calculated from known structures $[8,14]$. 


\subsection{Dynamic Viscosity Coefficient and Interfacial Tension Values of Different Extracts}

The dynamic viscosity coefficients of the four saponin extracts were evaluated (Figure 1a). There was no significant difference in the kinematic viscosity coefficient of the $30 \% \mathrm{EE}$ (376.8 MPa.s) and the CSCEE (415.6 MPas). Both the 50\% EE (925 MPa.s) and the 70\% EE (968.4 MPa.s) showed significantly higher kinematic viscosity coefficients than the seed cake ethanol extract.
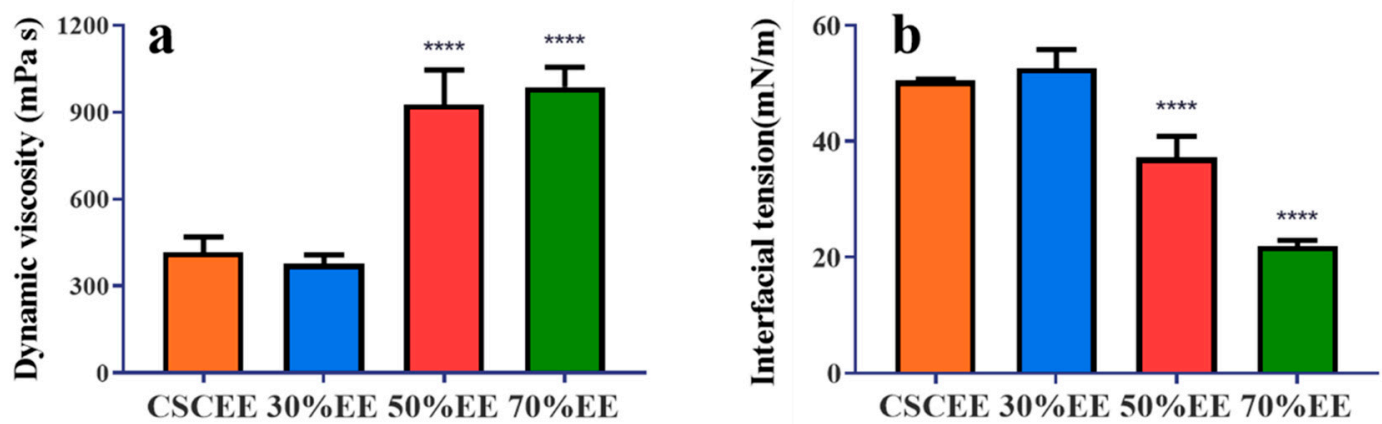

Figure 1. Viscosity coefficient and surface tension of the original seed cake extract and the different eluates from the AB- 8 macroporous resin. (a) Viscosity coefficient; (b) surface tension (CSCEE, C. oleifera seed cake ethanol extract; 30\%EE, 30\% ethanol eluate; $50 \% \mathrm{EE}, 50 \%$ ethanol eluate; $70 \% \mathrm{EE}, 70 \%$ ethanol eluate). Asterisks $\left(^{* * * *}\right)$ indicate significant differences compared with $C$. oleifera seed cake ethanol extract $(p<0.05), n=5$, average \pm SD. ANOVA with Tukey's HSD post-hoc test.

The interfacial tension values of the four saponin extracts were measured after 1000-fold dilution (Figure 1b). The results showed that the surface tension of the $70 \% \mathrm{EE}(21.86 \mathrm{mN} / \mathrm{m})$ was significantly lower than that of CSCEE $(50.412 \mathrm{mN} / \mathrm{m})$, the $30 \% \mathrm{EE}(52.64 \mathrm{mN} / \mathrm{m})$, and the $50 \% \mathrm{EE}(37.18 \mathrm{mN} / \mathrm{m})$.

\subsection{Toxicty of the Saponins on the Stomach of E. obliqua Larvae}

All saponin extracts were evaluated for toxicity to the stomach of E. obliqua larvae. No individuals died when fed distilled water (control) within $48 \mathrm{~h}$, so no adjustment for corrected mortality was necessary. The insecticidal effect of the $70 \% \mathrm{EE}\left(\mathrm{LC}_{50}=22.395 \mathrm{mg} / \mathrm{L}\right)$ was significantly higher than that of CSCEE $\left(\mathrm{LC}_{50}=49.100 \mathrm{mg} / \mathrm{L}, p<0.05\right), 30 \% \mathrm{EE}\left(\mathrm{LC}_{50}=53.239 \mathrm{mg} / \mathrm{L}, p<0.05\right)$ and $50 \% \mathrm{EE}$ $\left(\mathrm{LC}_{50}=45.287 \mathrm{mg} / \mathrm{L}, p<0.05\right)$ (Table 1$)$.

Table 1. Stomach toxicity (Ectropis obliqua larvae) of different saponin extracts from C. oleifera seed cake.

\begin{tabular}{ccccc}
\hline Pesticides & Toxicity Regression Equation ${ }^{\mathbf{a}}$ & $\mathbf{L C}_{\mathbf{5 0}} \mathbf{( \mathbf { m g } / \mathbf { L } ) ^ { \mathbf { b } }}$ & $\mathbf{9 5 \%}$ Confidence Interval & $\mathbf{R}^{\mathbf{c}}$ \\
\hline $\begin{array}{c}\text { C. oleifera seed cake } \\
\text { ethanol extract }\end{array}$ & $y=-6.69+4.12 x$ & 49.100 & $39.729-60.126$ & 0.874 \\
\hline $30 \%$ ethanol eluate & $y=-6.4+3.84 x$ & 53.239 & $42.799-66.291$ & 0.862 \\
$50 \%$ ethanol eluate & $y=-6.72+4.21 x$ & 45.287 & $36.527-55.263$ & 0.905 \\
$70 \%$ ethanol eluate & $y=-4.52+3.39 x$ & 22.395 & $16.489-28.338$ & 0.943 \\
\hline
\end{tabular}

a. Toxicity regression equation represents the relationship between $\log$ doses and lethality values. $\mathrm{b}$. $\mathrm{LC}_{50}$ represents lethal concentration $50 \%$, the dose required to kill half the members of a tested population after a specified test duration. c. $95 \%$ confidence interval of $\mathrm{LC}_{50}$.

\subsection{Contact Toxicity of the Camellia Saponins on E. obliqua Larvae}

Using the protocol for testing pesticides against Myzus persicae (Sulzer), the contact toxicity of the four saponin extracts on E. obliqua larvae were compared [15]. Overall, the toxicity of the four saponins was higher via contact than in the stomach (Table 2). The insecticidal activities were further evaluated by calculating the $\mathrm{LC}_{50}$ values of the four treatments on E. obliqua. The $\mathrm{LC}_{50}$ value of the $70 \% \mathrm{EE}$ was only $8.459 \mathrm{mg} / \mathrm{L}$, significantly lower (at $p<0.05$ ) than that of the CSCEE $\left(\mathrm{LC}_{50}=27.380 \mathrm{mg} / \mathrm{L}\right.$ ), the $30 \%$ $\mathrm{EE}\left(\mathrm{LC}_{50}=21.004 \mathrm{mg} / \mathrm{L}\right)$, and the $50 \% \mathrm{EE}\left(\mathrm{LC}_{50}=15.732 \mathrm{mg} / \mathrm{L}\right)$. 
Table 2. Contact toxicity (Ectropis obliqua larvae) of different saponin extracts from C. oleifera seed cake.

\begin{tabular}{|c|c|c|c|c|}
\hline Pesticides & Toxicity Regression Equation ${ }^{a}$ & $\mathrm{LC}_{50}(\mathrm{mg} / \mathrm{L})^{\mathrm{b}}$ & $95 \%$ Confidence Interval ${ }^{c}$ & $\mathbf{R}^{2}$ \\
\hline $\begin{array}{c}\text { C. oleifera seed cake } \\
\text { ethanol extract }\end{array}$ & $y=-5.00+3.43 x$ & 27.380 & $21.428-36.969$ & 0.977 \\
\hline $30 \%$ ethanol eluate & $y=-6.46+4.94 x$ & 21.004 & $16.848-25.238$ & 0.907 \\
\hline $50 \%$ ethanol eluate & $y=-3.75+3.14 x$ & 15.732 & $8.677-20.814$ & 0.834 \\
\hline $70 \%$ ethanol eluate & $y=-3.72+4.13 x$ & 8.459 & $3.564-11.646$ & 0.984 \\
\hline
\end{tabular}

a. Toxicity regression equation represents the relationship between log doses and lethality values. b. $\mathrm{LC}_{50}$ represents lethal concentration $50 \%$, the dose required to kill half the members of a tested population after a specified test duration. c. $95 \%$ confidence interval of $\mathrm{LC}_{50}$.

\subsection{Midgut of E. obliqua Larvae and Morphological Changes after Tea Saponin Treatment}

Larvae were treated for saponin for $24 \mathrm{~h}$, and the midgut was sectioned and observed by transverse staining. In the control group (Figure 2a), the midgut epithelium consisted of a single layer of digestive cells exhibiting a well-developed brush border and cytoplasm with acidophilic regions (Figure 2a, black arrow indicates example section). In contrast (Figure 2b), exposure of E. obliqua larvae to tea saponins resulted in a cell apex with a damaged brush border (Figure $2 b$, red arrow sections) and nucleus with decondensed chromatin and evident nucleoli (Figure $2 b$, blue arrow section). The intestinal wall was disrupted and had several holes (Figure $2 b$, green arrows).
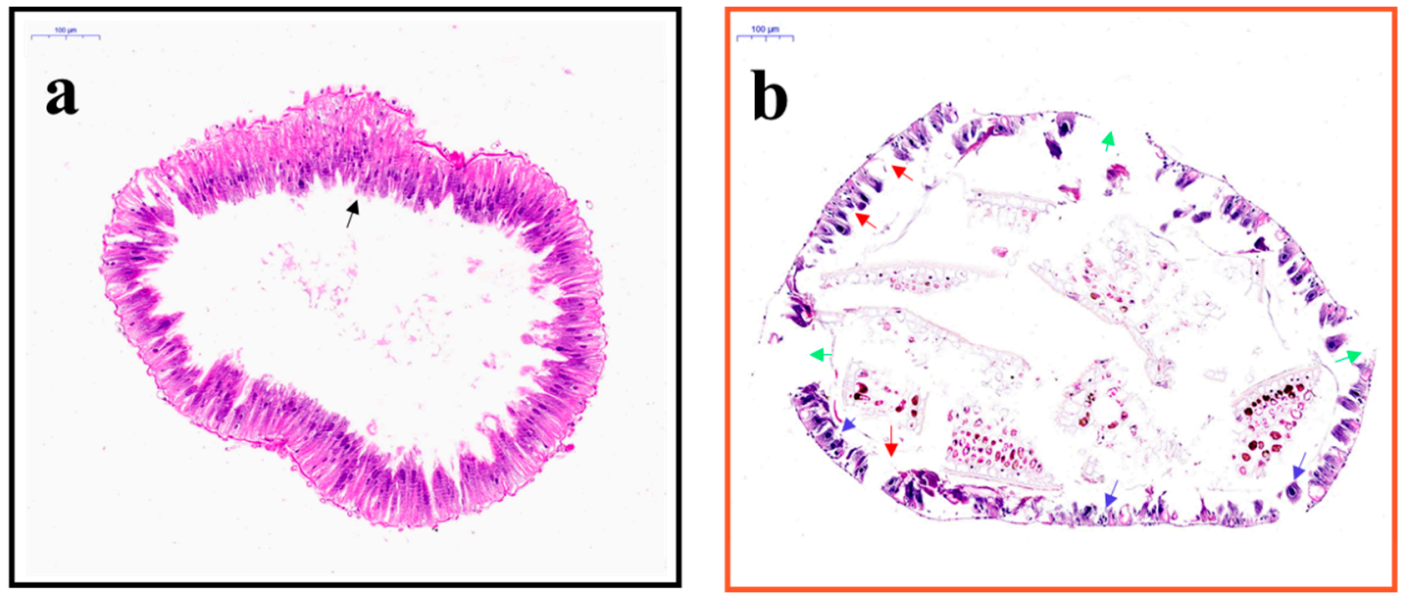

Figure 2. Cell staining of the intestinal tract of the E. obliqua larvae and the structural changes after (a) water treatment and (b) tea saponin treatment.

\subsection{Observation of the Epidermis of E. obliqua Larvae Treated with Tea Saponin by Electron Microscopy}

The effects of the two treatments on the epidermis of the E. obliqua larvae were observed by scanning electron microscopy (Figure 3). Compared with the control treatment (Figure 3a, panel a1), the epidermis layer of the E. obliqua larvae treated with the aqueous solution containing tea saponins became rough and formed severe wrinkles (Figure 3b, panel b1), and the trunk portion was dehydrated and shrunk (magnification at 100x). The waxy layer of the epidermis was ablated (at $5000 \times$, Figure 3b, panel b2, green arrow section) and voids appeared in the epidermis (Figure 3b, panel b3, red arrow section).

\subsection{Chitin Staining of E. obliqua Larvae and Morphological Changes after Tea Saponin Treatment}

In order to investigate the cause of the epidermal damage after tea saponin treatment, the chitin layer of the E. obliqua larvae was stained with lectin (Figure 4). Compared with the control (Figure 4a), the tea-saponin-treated E. obliqua showed thinning of the epidermal chitin and ablation (Figure $4 \mathrm{~b}$, yellow arrow), and the intestine (enclosed by the chitin layer) was ulcerated (Figure $4 \mathrm{~b}$, red arrow). 
a

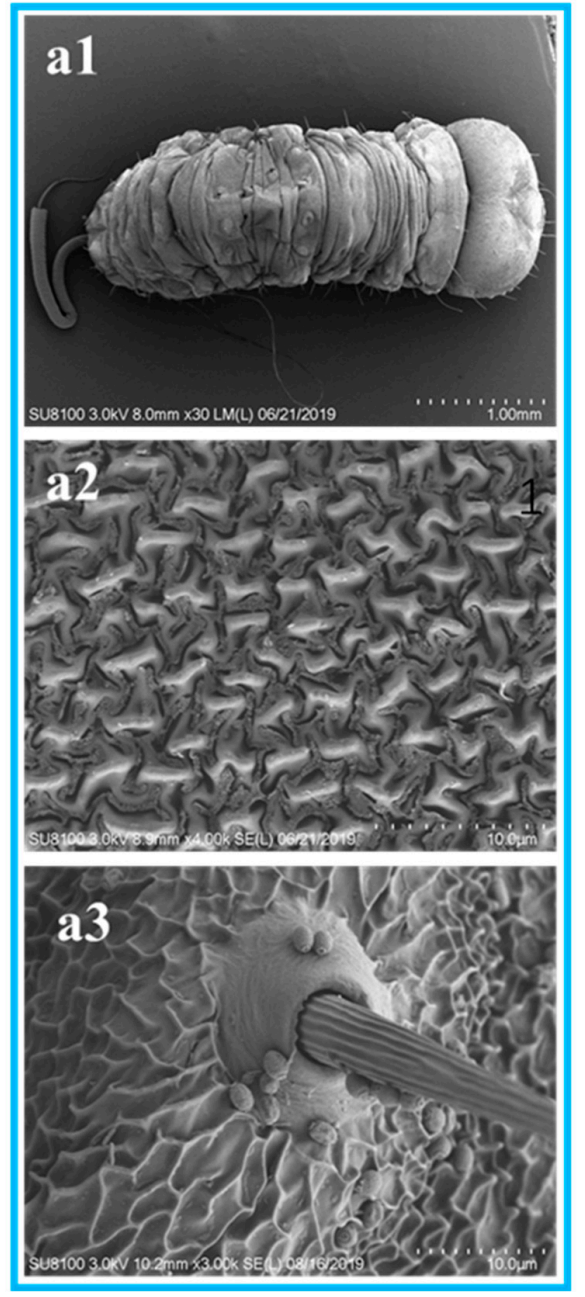

b

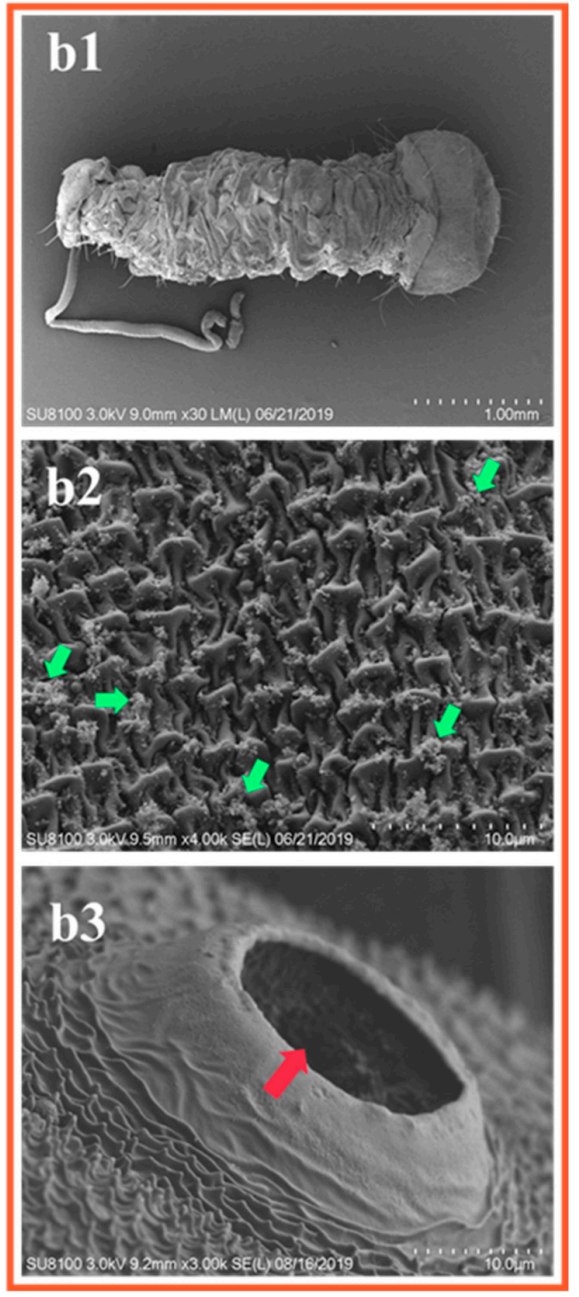

Figure 3. Scanning electron microscopy images of surfaces of E. obliqua larvae. (a) Control treatment at $100 \times(\mathrm{a} 1), 5000 \times(\mathrm{a} 2)$, and $5000 \times(\mathrm{a} 3)$ near the hairs. (b) Tea saponin treatment at $100 \times, 1000 \times$ and $5000 \times(\mathrm{b} 1, \mathrm{~b} 2$, and $\mathrm{b} 3$ respectively).
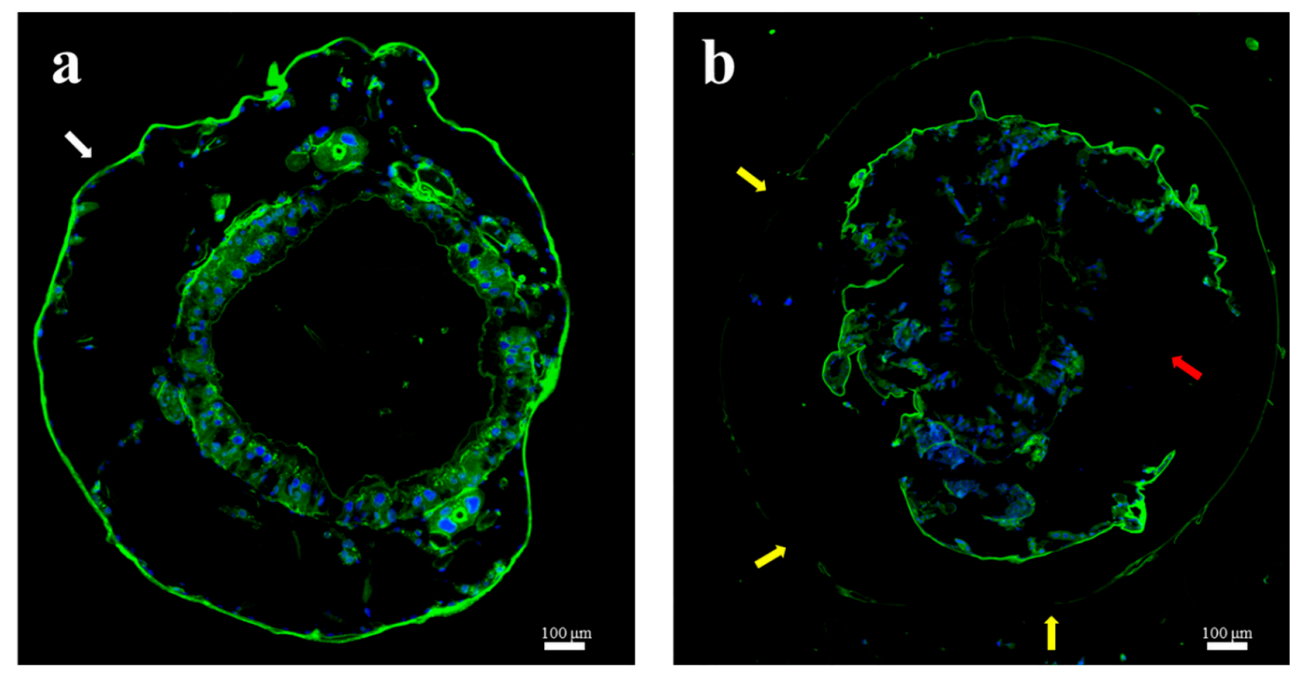

Figure 4. Chitin staining of E. obliqua larvae and midgut sections: (a) control; (b) tea saponin treatment). 


\section{Discussion}

In the search for novel plant-derived compounds for pest treatment in tea gardens, we found that a saponin extract from the same Camellia genus has advantages as an insect feeding deterrent and insecticide. In previous studies, tea saponins have been reported as natural, triterpenoid-derived compounds with insecticidal activity and resistance to insects [8,11]. Chen et al. (1996) demonstrated that a solution containing $25 \%$ active tea saponins significantly increased the larval mortality $(84 \%)$ of the cabbage butterfly (Pieris rapae Linne) [16]. In this study, the classic stomach toxicity test was used to evaluate stomach toxicity of tea saponins of different purities on E. obliqua larvae [17]. The results further verified that tea saponins have insecticidal properties and tea saponins with $99 \%$ purity showed 2.2 -fold higher stomach toxicity than did crude ethanol extract on E. obliqua larvae. The purity of tea saponins in the extract showed a good linear relationship with the insecticidal toxicity $\left(R^{2}=0.9865\right)$, which proved that tea saponins are the main insecticidal active ingredients in C. oleifera seed extract $[18,19]$.

In this study, tea-saponin-treated leaves caused severe damage to the midgut of E. obliqua larvae. The main functions of the midgut include the production of digestive enzymes and nutrient uptake of digested products [20-23]. The peritrophic matrix (PM) in the midgut of insects consists primarily of chitin and proteins and is thought to support digestion and provide protection from abrasive food particles and enteric pathogens [24]. The results indicate that tea saponins can cause physiological and morphological damage to midgut epithelial cells. After absorption of tea saponins, the cells exhibited vacuolization and vesicle release for energy detoxification. Excessive toxicity of tea saponins leads to smaller microvilli in the midgut and to cell death.

Evaluation of the contact toxicity of natural plant-derived extracts is important $[25,26]$. Some natural products have been reported to have direct contact toxicity on insects. For instance, linalool exhibited contact toxicity with an $\mathrm{LC}_{50}$ of $105.63 \mu \mathrm{g} / \mathrm{cm}^{2}$ against S. oryzae and Tribolium castaneum [27]. Wang et al. found that the essential oil from Z. purpureum rhizomes possessed strong contact toxicity against $T$. castaneum and Lasioderma serricorne adults, with $\mathrm{LD}_{50}$ values of 39.0 and $16.3 \mu \mathrm{g}$ per adult, respectively [28]. However, the contact toxicity of tea saponins has rarely been reported [29]. During these experiments, we found that an aqueous tea saponin solution can more easily adhere than just water to the back epidermis of E. obliqua. A high kinematic viscosity coefficient is beneficial to the effective adhesion of pesticides to the target insects and crops during spraying, and is positively correlated with the utilization rate of pesticides [30]. Our results are consistent with this, as the $70 \%$ EE saponin solution has a higher viscosity coefficient and a better contact toxicity against E. obliqua. This property could limit the movement of the larvae to some extent while increasing the effective retention time of the droplets on its epidermis.

The epidermis (exoskeleton) of E. obliqua is composed of a waxy layer and a chitin layer. The waxy later is the outermost layer of the epidermis and is highly hydrophobic. It is difficult for pesticides to penetrate into the skin or even the body. Therefore, it is important to evaluate the interfacial tension of the pesticide droplets. The lower the interfacial tension, the faster the pesticide droplets can spread on and penetrate the insect surface, thus improving the bioavailability of the pesticide [31,32]. In this experiment, the $70 \% \mathrm{EE}$ had the lowest interfacial tension and the strongest contact effect on E. obliqua. The purity of the tea saponin showed a good linear relationship with the interfacial tension $\left(R^{2}=0.9598\right)$. At the same time, the interfacial tension and contact toxicity also showed a good linear relationship $\left(R^{2}=0.9136\right)$. These data proved that tea saponins can effectively adhere to and penetrate into E. obliqua and that the high viscosity and low interfacial tension of tea saponins result in a higher bioavailability than some other natural products.

Some studies suggest that the waxy layer is the primary barrier that protects insects from external compounds [33]. The waxy layer prevents dryness, perceives the environment, and provides mechanical support and movement. In high-chlorine and octyl oil solutions, the waxy layer of the insect body is destroyed by adding a high-efficiency wax penetrant, so that the active ingredient penetrates the surface of the body and reaches the target site, thereby generating strong contact toxicity [34]. Some natural products have been found to disrupt the insect waxy layer, but the effects of saponins 
on insect waxy layers have rarely been reported [35]. In the present study (Figure 3), observation by scanning electron microscopy showed that a large area of ablation occurred in the waxy layer of the E. obliqua treated with tea saponins, resulting in the appearance of pores on the larvae and loss of water. The results revealed that tea saponins can cause higher toxicity during a short contact time. Tea saponins affect the water balance of the larvae by destroying the waxy layer on the surface, allowing penetration into the body and finally resulting in death due to water loss.

Many studies have shown that the thickening of the chitin layer is the main mechanism for insects to improve pesticide resistance. For example, a population of Anopheles gambiae originating from West Africa is resistant to pyrethroids and DDT due to a thickening of all chitin layers (exocuticle, mesocuticle and endocuticle) [36]. The proteins CPLCG3 (and G4) are expressed at higher levels in pyrethroid-resistant compared to susceptible mosquitoes [37,38]. Localization of these proteins in limbs and particularly in the endocuticle is consistent with the cuticle-thickening resistance mechanism [39]. Previous studies have shown that tea saponins can significantly induce chitinase activity in mycelial cells, causing chitin hydrolysis, resulting in increased cell membrane permeability as well as leakage of soluble proteins and reduction of reducing sugars [13,40]. Due to their amphiphilic structures, tea saponins easily interact with cholesterol substances and can interfere with the synthesis of ecdysteroids, thereby causing damage to the insect epidermis. In E. obliqua, chitin staining showed that the chitin content of the outer epidermis was significantly reduced after treatment with tea saponin and that the intestine disrupted after breakdown of the chitin in the intestinal tract. This proved that tea saponins can destroy not only the waxy layer on the surface of E. obliqua, but also the chitin layer both outside and in.

Together these results confirm that tea saponins have a strong insecticidal effect on the larvae of the tea plantation pest E. obliqua. The saponins can destroy the outer surfaces of the larvae by contact and can destroy the intestines following consumption. Through two different mechanisms of action, tea saponins can effectively control E. obliqua and protect tea trees. As a plant-derived insecticidal compound, tea saponins have a great potential to become a green pesticide, while future research will further explore the synergy of tea saponins with other types of pesticides (including biological pesticides) for the development of better pesticides.

\section{Materials and Methods}

\subsection{Plant Material and Extraction}

Camellia oleifera seed cake (20 kg, Yuzigui Oil Tea Co., Ltd., Huangshan, China) was dried, pulverized, and extracted twice with $70 \%$ ethanol at $60^{\circ} \mathrm{C}$ for $2 \mathrm{~h}$. The ethanol extract solution was filtered and dried with a spray dryer (LABMAQ, Riberão Preto-SP, Brazil, model MSDI 1.0). The extract powder was diluted to 3\% (w/v) with deionized water and batch-loaded onto a column (Chemical Co., Ltd., Xuzhou, Jiangsu, China,) containing AB-8 macroporous resin. After loading of the sample, the column was eluted with water (16 L) followed by increasing amounts of ethanol, beginning at $30 \%$ (16 L), 50\% (16 L), and then 70\% (32 L). The elutes were separately collected and dried for later analysis.

\subsection{Analytical Method}

The sulfuric-acid-ethanol color reaction was used for quality assessment of the tea saponin eluates. The ethanol extract of the tea saponin was diluted to $10 \mathrm{mg} / \mathrm{mL}$. Aliquots of the saponin solution were spotted on the TLC plates (pre-coated $20 \mathrm{~cm} \times 20 \mathrm{~cm}$ and $0.25 \mathrm{~mm}$ thickness, Anhui Liangchen Silicon Source Material Factory) using a capillary tube. Each sample-spotted sheet was put into a solvent system using toluene-ethyl-acetate-water $(4: 1: 0.7, v / v / v)$ as the mobile phase for a 5-min immersion. The plate was moved from developing cylinder and dried in an oven at $120^{\circ} \mathrm{C}$ for $3 \mathrm{~min}$. The plate was sprayed uniformly with $10 \%$ sulfuric acid-ethanol-vanillin solution, and then put on an iron plate at $105^{\circ} \mathrm{C}$ for $5 \mathrm{~min}$ for the color change. The reaction of tea saponins with sulfuric-acid-ethanol would appear purple, while flavonoids would be yellow. 
The purity of the tea saponin samples was further detected with HPLC according to the published method [14]. The oleiferasaponin C1 standard was accurately weighed ( $5 \mathrm{mg})$, sonicated with methanol, and diluted to $1 \mathrm{~mL}$ to yield a standard stock solution [41]. This was diluted to different concentrations with methanol to yield standard working solutions of 1000, 500, 200, 50, and $20 \mathrm{mg} / \mathrm{L}$. High-performance liquid chromatography (HPLC) (Agilent1260, Santa Clara, CA, USA) was used to detect the purity of the $C$. oleifera seed cake ethanol extract (water elution, CCEE), 30\% ethanol eluate (EE), 50\% EE, and $70 \%$ EE. The $70 \%$ EE was further evaluated by ultrahigh-performance chromatography coupled with electrospray ionization quadrupole time-of-flight mass spectrometry (UPLC-Q-TOF/MS), as described in [14].

\subsection{Interfacial Tension and Dynamic Viscosity Coefficient of the Different Extracts}

The viscosities of the extracts were first estimated to select the appropriate speed (12 RPM) and rotor size (L2) for the NDJ-8S digital viscometer (Shanghai Pingxuan Scientific Instrument Co., Ltd., Shanghai, China). The four extracts were diluted with deionized water 1000 times. After 2 min of equilibration at $25^{\circ} \mathrm{C}$, the measurement was repeated 5 times. Data are expressed in MPa.s.

The interfacial tension of the extract was determined using the maximum bubble method, with water as the constant for the liquid to be measured (DMPY-2C, Nanjing Nandawanhe Technology Co., Ltd., Nanjing, China). The end-point of the capillary was tangent to the water surface. The pesticide drop rate from the capillary was set to one drop every 5 to $10 \mathrm{~s}$. Each time a bubble escaped, the maximum pressure difference was recorded. Recordings were made for 10 to 20 groups to determine the average $\Delta \mathrm{P}_{\max }$ of water. The surface tension of the water at the experimental temperature was determined, and the instrument constant, $\mathrm{K}$, was calculated by the formula $\mathrm{K}=\Delta \mathrm{P}$ maxwater $/ \sigma_{\text {water }}$. To determine the surface tension of the sample, the test and capillary tubes were washed with the solution to be tested before an appropriate amount of sample was added to the test tube for measurement according to the instrument constant. The maximum pressure difference between 10 and 20 groups when jumping out of the bubble was recorded. Substitution of the average into the formula $\mathrm{K}=\Delta \mathrm{P}_{\text {maxwater }} / \sigma_{\text {water }}=\Delta \mathrm{P}_{\text {maxmeasured }} / \sigma_{\text {measured }}$ was used to calculate the surface tension.

\subsection{Bioassays}

\subsubsection{Evaluation of the Contact Toxicity of Camellia Saponins on E. obliqua}

A total of 2000 E. obliqua at the second instar stage were randomly divided into groups. The untreated (blank) group consisted of 80 E. obliqua. The remaining E. obliqua were treated with C. oleifera seed cake ethanol extract, $30 \% \mathrm{EE}, 50 \% \mathrm{EE}$, and $70 \% \mathrm{EE}$. Each pesticide treatment was diluted with deionized water to 8 different concentrations $(5,10,15,20,25,30,40$, and $50 \mathrm{mg} / \mathrm{L})$. Twenty E. obliqua larvae were tested as a group within the experiments, and three biological replicates were performed. A Burkard automated microtiter was used to drop $1 \mu \mathrm{L}$ of a treatment onto the back of each larvae. Mortality was observed $24 \mathrm{~h}$ after treatment and used to establish the respective concentration-response curves. The experiment was carried out in an artificial climate chamber (temperature $23 \pm 2{ }^{\circ} \mathrm{C}$, light:dark $=16: 8 \mathrm{~h}$, humidity $75 \% \mathrm{RH}$ ). The food was tea leaves (Shuchazao, Camellia sinensis) from the Agricultural Development Park of Anhui Agricultural University.

\subsubsection{Evaluation of the Stomach Toxicity of Camellia Saponins on E. obliqua}

The leaf-dip bioassay method described by Beloti et al. [42] and Liang et al. [43] was adopted to test the toxicity of tea saponins to second instar E. obliqua larvae. Eight tea saponins concentrations $(0.5,15,25,40,50,80,100$ and $120 \mathrm{mg} / \mathrm{mL})$ were assayed. Distilled water was used to prepare the dilutions. Tea leaf discs (diameter $4 \mathrm{~cm}$ ) were dipped for $20 \mathrm{~s}$ in one of the concentrations. The leaf discs were dried by placing them in a glass Petri dish (diameter $9 \mathrm{~cm}$ ). Leaf discs in the control group were dipped in distilled water as described above. Thirty E. obliqua larvae were starved for $24 \mathrm{~h}$ and then transferred to the glass Petri dish (two leaves per Petri dish). Three replicates were 
made for each concentration. Larvae were considered to be dead if they did not respond when lightly prodded with a brush. Mortality was observed $48 \mathrm{~h}$ after treatment and used to establish the respective concentration-response curves.

\subsubsection{Midgut Slices of E. obliqua}

The E. obliqua were fed using a leaf soaked with $50 \mathrm{ppm}$ tea saponins according to the method of evaluation of stomach toxicity. After $24 \mathrm{~h}$, the larvae were anesthetized in diethyl ether before the intestines were taken out and fixed in Bouns solution for $12 \mathrm{~h}$. Following this, the intestines were desiccated and diaphanized in alcohol/xylene (1:1) according to Michalany (1980). After placing in paraffin, the samples were sliced in $7-\mu \mathrm{m}$ sections and stained with hematoxylin-eosin. Morphological alterations of the mid-gut of treated larvae were desiccated and compared to the tissues taken from the control group that were fed untreated leaves and prepared in the same way.

\subsubsection{Scanning Electron Microscopy}

Water and tea saponins in water $(10 \mathrm{mg} / \mathrm{L} ; 1 \mu \mathrm{L}$ either solution) were separately dropped onto the back of the E. obliqua larvae using a Burkard automated microtiter. After $24 \mathrm{~h}$, samples were fixed in $2.5 \%$ glutaraldehyde overnight and then washed 4 times with phosphate buffer for $30 \mathrm{~min}$. The samples were dehydrated in a stepwise manner for $30 \mathrm{~min}$ in 30\%, 50\%, 70\%, 80\%, 90\%, and 100\% alcohol. Isoamyl acetate was replaced twice for $30 \mathrm{~min}$ each time. The samples were dried under vacuum $\left(0.1 \mathrm{mbar}\right.$, temperature $\left.-42{ }^{\circ} \mathrm{C}\right)$. Samples were adhered to the sample plate with conductive tape and observed using a scanning electron microscope (Hitachi SU-8100, Tokyo, Japan) to evaluate the surface structure, shape, and size characteristics of the E. obliqua. The SEM was carried out at magnification range of 100-5000×.

\subsubsection{Chitin Staining of E. obliqua Sections}

E. obliqua larvae (treated with saponin as described in Section 4.4.4) were fixed overnight in $2.5 \%$ glutaraldehyde, washed three times for $5 \mathrm{~min}$ each with $150 \mathrm{mM} \mathrm{NaCl}, 10 \mathrm{mM} \mathrm{Na} \mathrm{HPO}_{4}$, and $10 \mathrm{mM}$ $\mathrm{NaH}_{2} \mathrm{PO} 4$ (pH 7.2), and then fixed in 30\% sucrose glutaraldehyde for $8 \mathrm{~h}$. The anterior of the larvae was embedded in optimum cutting temperature compound (O.C.T. Sakura, Torrance, CA, USA). Sections $(8 \mu \mathrm{m})$ were cut using a Leica CM1950 microtome (Leica Microsystems, Wetzlar, Germany) and washed three times for $10 \mathrm{~min}$ in PBS (137 mM NaCl, $2.7 \mathrm{mM} \mathrm{KCl}, 10 \mathrm{mM} \mathrm{Na} 2 \mathrm{HPO}_{4}, 2 \mathrm{mM} \mathrm{KH}_{2} \mathrm{PO}_{4}, \mathrm{pH}$ 7.4). The sections were incubated with DAPI dihydrochloride solution (Beyotime), washed three times for 10 min with PBST (PBS containing 0.1\% Tween), incubated with wheat germ agglutinin FITC-labeled (WAG) (Sigma), and then washed three times for $10 \mathrm{~min}$ with PBST. The slides were photographed using an Olympus BX51 microscope.

\subsection{Data Analysis}

All experiments were carried out at least three times and the data were reported as means and standard deviations. Statistical analyses were performed using SPSS 20.0 (IBM Corp Version 20.0, IBM SPSS Statistics for Windows; IBM, Armonk, NY, USA) and Prism 5 (GraphPad Software, La Jolla, CA, USA) software. Significance was analyzed by the least significant difference (LSD) test with a $95 \%$ confidence level $(p<0.05)$.

Supplementary Materials: The following are available online, Table S1. The molecular formula of the main compound in 70\% ethanol solution and Peak identity (LC/TOF-MS). Table S2. Estimated molecular weights for the main compound in 70\% ethanol solution (LC/TOF-MS). Figure S1. Thin layer chromatography analysis of tea saponin components in different eluents. Figure S2. Liquid chromatographic analysis of tea saponins in different eluents. Figure S3. Total ion chromatogram of saponin in 70\% ethanol solution (LC/TOF-MS).

Author Contributions: Conceived the project, R.H.; X.W.; H.C.; C.P.; performed the experiments, collected and analyzed data, C.C.; supervised and provided biological material for assay on E. obliqua, Y.Y.; T.Z.; assist in 
insecticidal tests, K.Z.; writing—Reviewed manuscript by all authors. Each author approved the final version of the manuscript of submission.

Funding: This work was supported by the National Natural Scientific Foundation of China (No. 31772076), the National Key Research \& Development Program (2016YFD0200900) of China, and project of "The control technical application of tea quality and Safety, Anhui Provinical Key Program for Research and Development (2019)".

Acknowledgments: The authors are grateful for the comments by Drs. Liwei Gu and Janine Spies at the University of Florida.

Conflicts of Interest: The authors declare no conflict of interest.

\section{References}

1. Malongane, F.; Mcgaw, L.J.; Mudau, F.N. The synergistic potential of various teas, herbs and therapeutic drugs in health improvement: A review. J. Sci. Food Agric. 2017, 97, 4679-4689. [CrossRef] [PubMed]

2. Sinu, P.A.; Mandal, P.; Banerjee, D.; Mallick, S.; Talukdar, T.; Pathak, S.K. Moth pests collected in light traps of tea plantations in North East India: Species composition, seasonality and effect of habitat type. Curr. Sci. 2013, 104, 646-651.

3. Ma, L.; Li, Z.-Q.; Bian, L.; Cai, X.-M.; Luo, Z.-X.; Zhang, Y.-J.; Chen, Z.-M. Identification and comparative study of chemosensory genes related to host selection by legs transcriptome analysis in the tea geometrid Ectropis obliqua. PLoS ONE 2016, 11, e0149591. [CrossRef] [PubMed]

4. Wang, D.; Chen, L. Research of resistance mechanism to Ectropis oblique by tea plant. J. Tea Sci. 2014, 34, 541-547.

5. Feng, J.; Tang, H.; Chen, D.; Li, L. Monitoring and risk assessment of pesticide residues in tea samples from China. Hum. Ecol. Risk Assess. Int. J. 2015, 21, 169-183. [CrossRef]

6. Zhao, H.X.; Zhao, S.C.; Deng, L.G.; Mao, J.S.; Guo, C.Y.; Yang, G.S.; Lu, X.; Aboul-Enein, H.Y. Rapid Determination of Organonitrogen, Organophosphorus and Carbamate Pesticides in Tea by Ultrahigh-Performance Liquid Chromatography-Tandem Mass Spectrometry (UPLC-MS/MS). Food Anal. Methods 2013, 6, 497-505. [CrossRef]

7. Fang, Q.; Shi, Y.; Cao, H.; Tong, Z.; Xiao, J.; Liao, M.; Wu, X.; Hua, R. Degradation Dynamics and Dietary Risk Assessments of Two Neonicotinoid Insecticides during Lonicera japonica Planting, Drying, and Tea Brewing Processes. J. Agric. Food Chem. 2017, 65, 1483-1488. [CrossRef]

8. Cui, C.; Zong, J.; Sun, Y.; Zhang, L.; Ho, C.-T.; Wan, X.; Hou, R. Triterpenoid saponins from the genus Camellia: Structures, biological activities, and molecular simulation for structure-Activity relationship. Food Funct. 2018, 9, 3069-3091. [CrossRef]

9. Wu, H. Study on Influencing Factors and Trend Prediction of Camellia Industry Development in China; Chinese Academy of Forestry: Beijing, China, 2017.

10. Dolma, S.K.; Sharma, E.; Gulati, A.; Reddy, S.E. Insecticidal activities of tea saponin against diamondback moth, Plutella xylostella and aphid, Aphis craccivora. Toxin Rev. 2018, 37, 52-55. [CrossRef]

11. Lin, S.; Chen, Y.; Bai, Y.; Cai, H.; Wei, H.; Tian, H.; Zhao, J.; Chen, Y.; Yang, G.; Gu, X.; et al. Effect of Tea Saponin-treated host plants on activities of antioxidant enzymes in larvae of the Diamondback Moth Plutella xylostella (Lepidoptera: Plutellidae). Env. Entomol. 2018, 47, 749-754. [CrossRef]

12. Cai, H.; Bai, Y.; Wei, H.; Lin, S.; Chen, Y.; Tian, H.; Gu, X.; Murugan, K. Effects of tea saponin on growth and development, nutritional indicators, and hormone titers in diamondback moths feeding on different host plant species. Pestic. Biochem. Physiol. 2016, 131, 53-59. [CrossRef] [PubMed]

13. Chaieb, I. Saponins as insecticides: A review. Tunis. J. Plant Prot. 2010, 5, 39-50.

14. Zhang, X.-F.; Yang, S.-L.; Han, Y.-Y.; Zhao, L.; Lu, G.-L.; Xia, T.; Gao, L.-P. Qualitative and quantitative analysis of triterpene saponins from tea seed pomace (Camellia oleifera abel) and their activities against bacteria and fungi. Molecules 2014, 19, 7568-7580. [CrossRef] [PubMed]

15. Devonshire, A.L.; Moores, G.D. A carboxylesterase with broad substrate specificity causes organophosphorus, carbamate and pyrethroid resistance in peach-potato aphids (Myzus persicae). Pestic. Biochem. Physiol. 1982, 18, 235-246. [CrossRef]

16. Chen, S.; Li, G.; Lai, J.; Li, X.; Zhang, Y. Study of tea saponin TS-D insecticidal effects on cabbage butterfly. Plant Prot. 1996, 22, 27-28. 
17. Zeng, C.; Wu, L.; Zhao, Y.; Yun, Y.; Peng, Y. Tea saponin reduces the damage of Ectropis obliqua to tea crops, and exerts reduced effects on the spiders Ebrechtella tricuspidata and Evarcha albaria compared to chemical insecticides. PeerJ 2018, 6, e4534. [CrossRef] [PubMed]

18. Chaicharoenpong, C.; Petsom, A. Quantitative thin layer chromatographic analysis of the saponins in tea seed meal. Phytochem. Anal. 2009, 20, 253-255. [CrossRef]

19. Li, N.; Ma, Z.-J.; Chu, Y.; Wang, Y.; Li, X. Phytochemical analysis of the triterpenoids with cytotoxicity and QR inducing properties from the total tea seed saponin of Camellia sinensis. Fitoterapia 2013, 84, 321-325. [CrossRef]

20. Snodgrass, R.E. The abdominal mechanisms of a grasshopper. Smithson. Misc. Collect. 1935, 94, 6.

21. Akai, H.; Kimura, K.; Kiuchi, M.; Shibukawa, A. Effects of anti-juvenoid treatment on cocoon and cocoon filaments in Bombyx mori. J. Sericultural Sci. Jpn. 1984, 53, 545-546.

22. Serrão, J.E.; Da Cruz-Landim, C. Gut structures in adult workers of necrophorous Neotropical stingless bees (Hymenoptera: Apidae: Meliponinae). Entomol. Gen. 1995, 19, 261-265. [CrossRef]

23. Cavalcante, V.; da Cruz-Landim, C. Types of cells present in the midgut of the insects: A review. Nat.-Sao Paulo. 1999, 24, 19-40.

24. Agrawal, S.; Kelkenberg, M.; Begum, K.; Steinfeld, L.; Williams, C.E.; Kramer, K.J.; Beeman, R.W.; Park, Y.; Muthukrishnan, S.; Merzendorfer, H. Two essential peritrophic matrix proteins mediate matrix barrier functions in the insect midgut. Insect Biochem. Mol. Biol. 2014, 49, 24-34. [CrossRef] [PubMed]

25. Bartlett, B.R. The contact toxicity of some pesticide residues to hymenopterous parasites and coccinellid predators. J. Econ. Entomol. 1963, 56, 694-698. [CrossRef]

26. Thompson, H.M. Assessing the exposure and toxicity of pesticides to bumblebees (Bombus sp.). Apidologie 2001, 32, 305-321. [CrossRef]

27. Cao, J.Q.; Guo, S.S.; Wang, Y.; Pang, X.; Geng, Z.F.; Du, S.S. Toxicity and repellency of essential oil from Evodia lenticellata Huang fruits and its major monoterpenes against three stored-product insects. Ecotoxicol. Environ. Saf. 2018, 160, 342-348. [CrossRef]

28. Wang, Y.; You, C.X.; Yang, K.; Wu, Y.; Chen, R.; Zhang, W.J.; Liu, Z.L.; Du, S.S.; Deng, Z.W.; Geng, Z.F. Bioactivity of Essential Oil of Zingiber purpureum Rhizomes and Its Main Compounds against Two Stored Product Insects. J. Econ. Entomol. 2015, 108, 925-932. [CrossRef]

29. Attia, S.; Grissa, K.L.; Lognay, G.; Bitume, E.; Hance, T.; Mailleux, A.C. A review of the major biological approaches to control the worldwide pest Tetranychus urticae (Acari: Tetranychidae) with special reference to natural pesticides. J. Pest Sci. 2013, 86, 361-386. [CrossRef]

30. Gil, Y.; Sinfort, C. Emission of pesticides to the air during sprayer application: A bibliographic review. Atmos. Environ. 2005, 39, 5183-5193. [CrossRef]

31. Wang, B.; Song, J.; Zeng, A.; Liu, Y.; Zhang, J.; He, X. Effects of formulations and surfactants on the behavior of pesticide liquid spreading in the plant leaves. Chin. J. Pestic. Sci. 2012, 14, 334-340.

32. Kirkwood, R.C. Recent developments in our understanding of the plant cuticle as a barrier to the foliar uptake of pesticides. Pestic. Sci. 1999, 55, 69-77. [CrossRef]

33. Balabanidou, V.; Grigoraki, L.; Vontas, J. Insect cuticle: A critical determinant of insecticide resistance. Curr. Opin. Insect Sci. 2018, 27, 68-74. [CrossRef] [PubMed]

34. Luzhen, W. Test on insecticidal effect of high chlorine and octyl oil. Agric. Technol. Equip. 2014, 22, 50-51.

35. Dayan, F.E.; Cantrell, C.L.; Duke, S.O. Natural products in crop protection. Bioorganic Med. Chem. 2009, 17, 4022-4034. [CrossRef] [PubMed]

36. Yahouédo, G.A.; Chandre, F.; Rossignol, M.; Ginibre, C.; Balabanidou, V.; Mendez, N.G.A.; Pigeon, O.; Vontas, J.; Cornelie, S. Contributions of cuticle permeability and enzyme detoxification to pyrethroid resistance in the major malaria vector Anopheles gambiae. Sci. Rep. 2017, 7, 11091. [CrossRef] [PubMed]

37. Vontas, J.; David, J.P.; Nikou, D.; Hemingway, J.; Christophides, G.; Louis, C.; Ranson, H. Transcriptional analysis of insecticide resistance in Anopheles stephensi using cross-species microarray hybridization. Insect Mol. Biol. 2007, 16, 315-324. [CrossRef]

38. Awolola, T.; Oduola, O.; Strode, C.; Koekemoer, L.; Brooke, B.; Ranson, H. Hygiene, Evidence of multiple pyrethroid resistance mechanisms in the malaria vector Anopheles gambiae sensu stricto from Nigeria. Trans. $R$. Soc. Trop. Med. Hyg. 2009, 103, 1139-1145. [CrossRef] 
39. Vannini, L.; Reed, T.W.; Willis, J.H. Temporal and spatial expression of cuticular proteins of Anopheles gambiae implicated in insecticide resistance or differentiation of M/S incipient species. Parasites Vectors 2014, 7, 24. [CrossRef]

40. Yang, X.; Zhang, H. Synergistic interaction of tea saponin with mancozeb against Pestalotiopsis theae. Crop Prot. 2012, 40, 126-131. [CrossRef]

41. Zong, J.; Wang, R.; Bao, G.; Ling, T.; Zhang, L.; Zhang, X.; Hou, R. Novel triterpenoid saponins from residual seed cake of Camellia oleifera Abel. show anti-proliferative activity against tumor cells. Fitoterapia 2015, 104, 7-13. [CrossRef]

42. Beloti, V.H.; Alves, G.R.; Araújo, D.F.D.; Picoli, M.M.; de Andrade Moral, R.; Demétrio, C.G.B.; Yamamoto, P.T. Lethal and sublethal effects of insecticides used on citrus, on the ectoparasitoid Tamarixia radiata. PLoS ONE 2015, 10, e0132128. [CrossRef] [PubMed]

43. Liang, P.; Gao, X.W.; Zheng, B.Z. Genetic basis of resistance and studies on cross-resistance in a population of diamondback moth, Plutella xylostella (Lepidoptera: Plutellidae). Pest Manag. Sci. Former. Pestic. Sci. 2003, 59, 1232-1236. [CrossRef] [PubMed]

Sample Availability: Samples of the compounds are available from the authors.

(C) 2019 by the authors. Licensee MDPI, Basel, Switzerland. This article is an open access article distributed under the terms and conditions of the Creative Commons Attribution (CC BY) license (http://creativecommons.org/licenses/by/4.0/). 